\title{
EMBRIOPATÍAS ASOCIADAS AL USO DE MISOPROSTOL
}

\author{
Andrea Osorio O., Juan Guillermo Rodríguez A., Oscar Pizarro R., Axel Paredes V., Susana \\ Aguilera $P$.
}

Centro de Referencia Perinatal Oriente (CERPO), Servicios y Departamento de Obstetricia y Ginecología y Neonatología, Hospital Santiago Oriente "Dr. Luis Tisné Brousse", Campus Oriente, Facultad de Medicina, Universidad de Chile.

\section{RESUMEN}

Se presenta un caso clínico de síndrome de Moebius y artrogriposis asociado al uso de misoprostol durante el primer trimestre de gestación.

\section{PALABRAS CLAVES: Misoprostol, síndrome de Moebius, artrogriposis}

\section{SUMMARY}

We present a case of Moebius syndrome and arthrogriposis associated to the use of misoprostol during the first trimester of gestation.

\section{KEY WORDS: Misoprostol, Moebius syndrome, arthrogriposis}

\section{INTRODUCCIÓN}

La artrogriposis múltiple congénita (AMC) se define como una condición no progresiva, de causa desconocida, caracterizada por la presencia de contracturas y deformidades articulares, debido a causas miogénicas y neurogénicas, que se expresan principalmente como hipomotilidad o acinesia fetal $(1,2)$. Es una entidad infrecuente, estimándose una incidencia de 1 en 3000-10000 embarazos. No existe diferencia racial o asociada al sexo del feto y sólo en $30 \%$ de los casos se encuentra una causa genética (3).

La fisiopatología de esta condición es relativamente desconocida y se caracteriza por la disminución de los movimientos fetales durante la gestación. Dentro de las causas fetales se incluyen alteraciones musculares, neurogénicas y/o del tejido conectivo, así como limitaciones mecánicas al movimiento debido a oligohidroamnios, malformaciones uterinas, bridas amnióticas, miomas uterinos, etc. Como causas maternas pueden men- cionarse las infecciosas, consumo de drogas como misoprostol $u$ otras, trauma y otras enfermedades maternas.

Por otro lado, el síndrome de Moebius (SM) corresponde a una diplejia facial congénita no progresiva y restricción de movimientos oculares laterales que se caracteriza por hipomimia, más evidente en ojos y en la sonrisa. Generalmente se presenta en forma bilateral y puede asociarse a parálisis de distintos nervios craneales (NC) como el oculomotor, trigémino, glosofaríngeo y vago. Se ha relacionado con malformaciones craneofaciales y de otras zonas del cuerpo. Dentro de las malformaciones craneofaciales podemos encontrar micrognatia, úvula bífida, epicanto y paladar hendido (4). Otras malformaciones asociadas son equinovaro y sindactilia (5). La etiología del SM se ha relacionado a múltiples causas, tanto genéticas como ambientales, que tendrían como resultado una ausencia congénita del núcleo del abducens (NC VI) y facial (NC VII). Dentro de los factores ambientales relacionados al SM se ha menciona- 
do la exposición durante el período de gestación a sustancias tales como cocaína, alcohol, talidomida y misoprostol $(6,7)$.

El objetivo de esta comunicación es presentar un caso clínico de síndrome de Moebius y artrogriposis, asociados al uso de misoprostol en las primeras etapas de la gestación como práctica para la interrupción del embarazo.

\section{CASO CLÍNICO}

Paciente de 22 años, primigesta, sin antecedentes mórbidos de importancia. Es derivada cursando 26+3 semanas de gestación al Centro de Referencia Perinatal Oriente (CERPO) desde un Consultorio del Área Oriente de Santiago con el diagnóstico de pie bot bilateral y manos en garra (Figura 1).
En CERPO se realiza ecotomografía obstétrica que confirma el diagnóstico de manos en garra y pie bot bilateral. Además se diagnostica polihidroamnios (PHA) moderado y crecimiento adecuado para la edad gestacional.

La paciente se controla periódicamente con ecotomografías obstétricas seriadas, sin cambios en el diagnóstico (Tabla I), asimismo refirió escasos movimientos fetales durante todo el embarazo.

A las $32,34+3$ y 37 semanas de gestación se realizó doppler obstétrico por diagnóstico de restricción del crecimiento fetal, resultando siempre dentro de rangos normales. La ecocardiografía fetal a las 29 semanas fue normal. El cariograma fetal efectuado de sangre fetal obtenida por cordocentesis fue normal $(46, \mathrm{XX})$. Durante el mismo procedimiento se controla hemograma fetal.

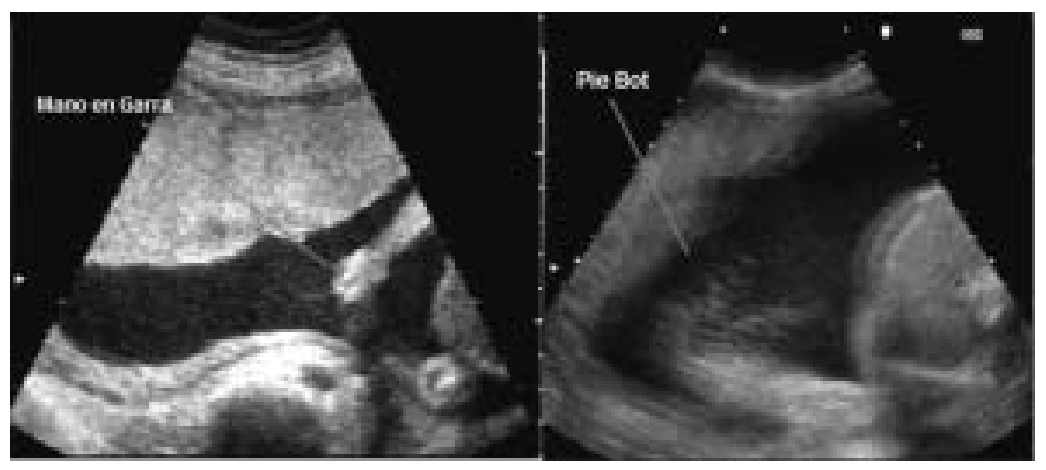

Figura 1. Manos en garra y pie Bot.

Tabla I

CONTROL ECOGRÁFICO OBSTÉTRICO SERIADO

\begin{tabular}{lccr}
\hline Controles & & 32 sem & 36 sem \\
Edad Gestacional & 26 sem & 81 & 86 \\
\hline Diámetro biparietal & 67 & 98 & 106 \\
DFO & 84 & 38 & 41 \\
Hemisferio & 29 & 8 & 7 \\
Ventrículo anterior & 3,5 & 8 & 7 \\
Ventrículo posterior & 4,4 & 35 & 42 \\
Cerebelo & 25 & 228 & 260 \\
CA & 193 & 56 & 65 \\
Longitud fémur & 48 & 39 & 44 \\
Talla & 35 & 1284 & 1953 \\
Peso fetal estimado & 779 & $10-25$ & $<5$ \\
Percentil & 25 & 300 & 327 \\
ILA & 218 & Artrogriposis. PHA marcado. & Artrogriposis. PHA marcado. \\
Conclusión & PHA moderado. Manos en & RCIU. Manos en garra. \\
& garra. Pie Bot bilateral. & RCIU. Manos en garra. & Pie Bot bilateral.
\end{tabular}

DFO: diámetro fronto-occipital. CA: circunferencia abdominal. PHA: polihidroamnios. RCIU: restricción del crecimiento intrauterino. ILA: índice líquido amniótico. 
El manejo incluyó consejería y manejo psicológico pre y post-natal por psicóloga de la Unidad.

A las 36 semanas de gestación, una monitorización fetal es sugerente de hipoxia crítica fetal, por lo que se decide interrupción del embarazo por cesárea, obteniéndose un recién nacido pretérmino de sexo femenino, pequeño para la edad gestacional (RNPTPEG). En la atención neonatal inmediata presenta asfixia perinatal severa (Apgar 2-5 al 1 y 5 minutos), trismus importante que dificulta la intubación y una fisura en $U$ invertida de paladar blando. Se realiza conexión inmediata a ventilación mecánica por no presentar actividad respiratoria. Peso: $1.685 \mathrm{~g}$, talla: $42,5 \mathrm{~cm}$, perímetro cefálico: $32,5 \mathrm{~cm}$.

Al examen físico destaca un RNPTPEG severo, sin movimientos respiratorios ni de extremidades, espontáneos y/o provocados. Con mirada fija, ojos abiertos, fascie menuda con desproporción cráneo facial, trismus y fisura del paladar ya descritos. Pabellones algo displásicos, mayor a derecha (Figura 2). Destaca además la presencia de manos en "garra" (campodactilia) con índices en extensión metacarpo-falángica proximal y flexión distal, pie derecho en posición tálipe equinovaro no reductible y pie izquierdo sólo con superposición de segundo ortejo sobre el primero (Figura 3). Evoluciona sin respiración autónoma, con total dependencia del ventilador, y sólo a los 10 días esboza movimientos de extremidades.

Reinterrogada la madre, refiere uso vaginal de 3 comprimidos de misoprostol alrededor de las 8 semanas de gestación.

En estudio postnatal destaca: radiografía tóracoabdominal con escoliosis de concavidad izquierda y vértebras conservadas (Figura 4), ultrasonido

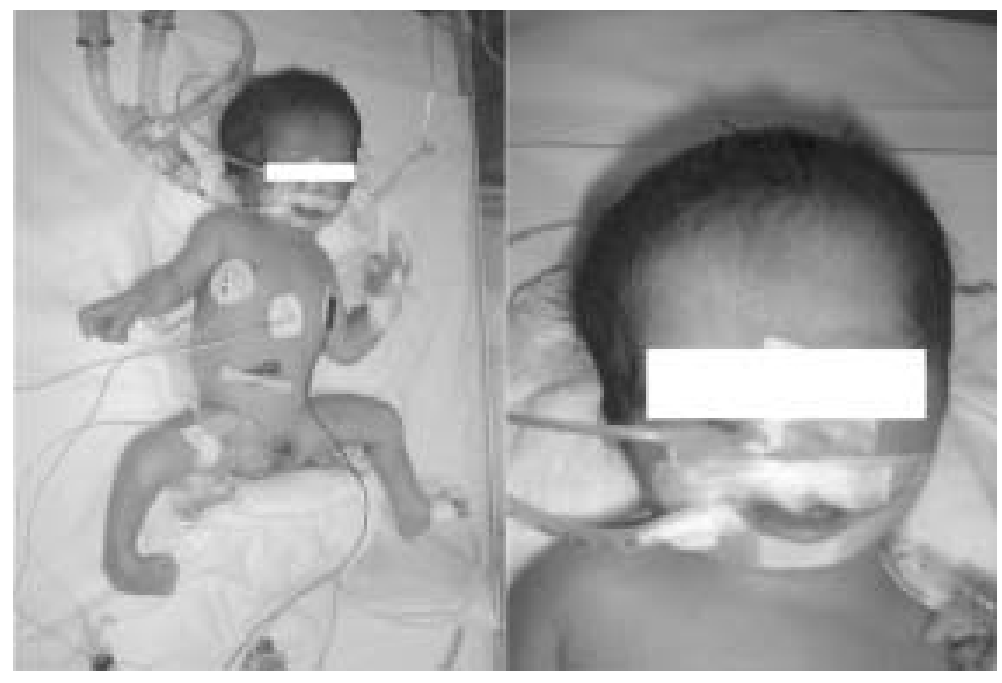

Figura 2. Síndrome de Moebius, artrogriposis.

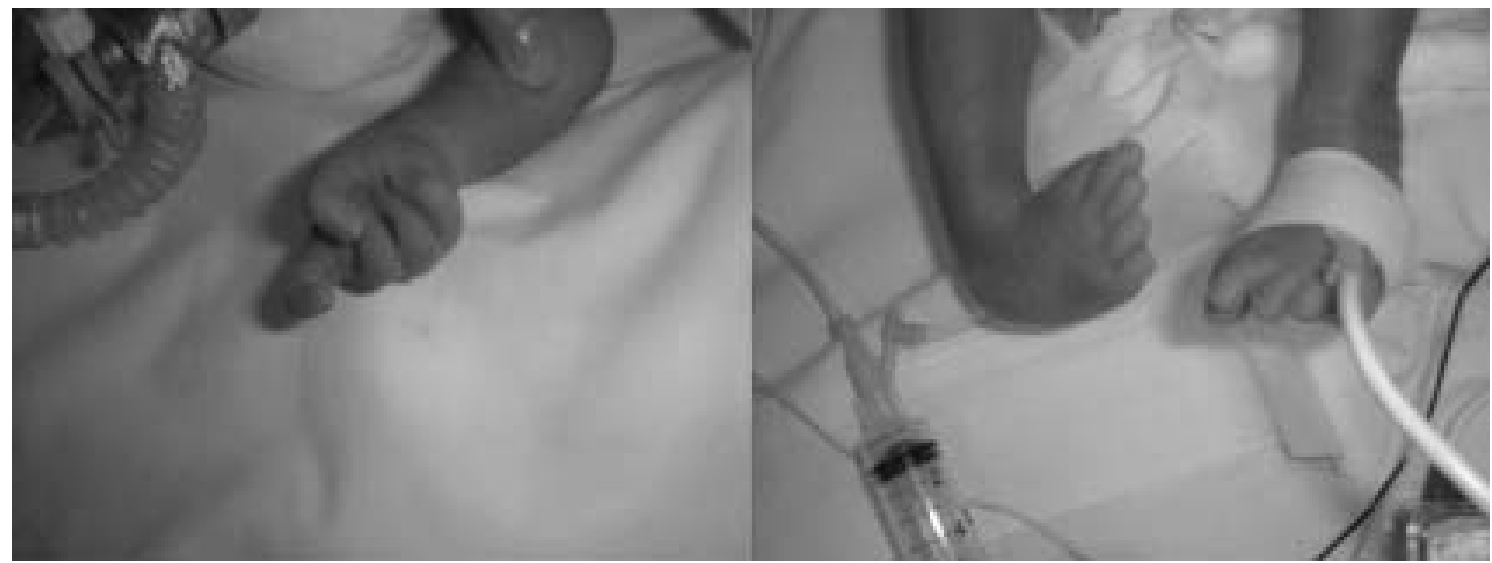

Figura 3. Manos en "garra" con índices en extensión metacarpo-falángica proximal y flexión distal. Pie tálipe equinovaro derecho no reductible con superposición de ortejo segundo sobre el mayor. 


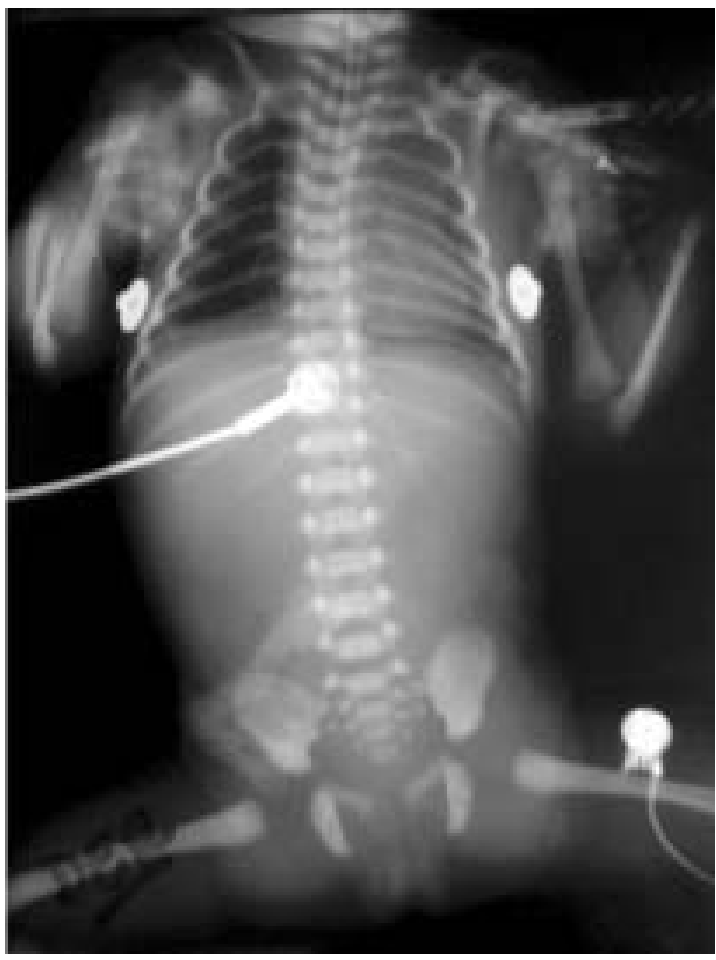

Figura 4. Radiografía tóraco-abdominal. Escoliosis de concavidad izquierda y vértebras conservadas.

encefálico dentro de lo normal, ultrasonido renal reveló ambos riñones lobulados, de aspecto inmaduro. La ecocardiografía realizada durante el primer mes de vida fue normal.

La evaluación neurológica a los 13 días de vida fue informada como: hipotonía axial con artrogriposis distal y de articulación témporo-maxilar; discreta movilización de las 4 extremidades, venciendo gravedad las superiores; diplejia facial periférica; parálisis VI par bilateral; parálisis IX-X. Conclusión: cuadro clínico compatible con síndrome de Moebius más artrogriposis.

A los 2 meses de vida continuaba con total dependencia a ventilación mecánica y sólo esbozo de movimiento de extremidades. A los 81 días de vida presenta deterioro respiratorio secundario a atelectasias bilaterales y hemograma sugerente de infección, atribuíble a bronconeumonia intrahospilataria, por lo que se trató 10 días con cloxacilina-amikacina, sin identificación del germen. Posteriormente evoluciona con atelectasias fluctuantes. Se presentó a comité de ética del hospital concluyéndose que no hay argumentos para abandonar su terapia de mantención incluyendo la ventilación mecánica. A los 95 días de vida presentó crisis convulsivas tónico clónicas que se yugularon con fenobarbital, indicándose completar estudio neurológico con TAC cerebral y EEG. Por su dependencia ventilatoria se traslada al Hospital Luis Calvo Mackenna para realizar el estudio, falleciendo a los pocos días por insuficiencia respiratoria.

\section{DISCUSIÓN}

El misoprostol es un análogo de la prostaglandina $E 1$, el cual inicialmente fue comercializado para la prevención y tratamiento de úlceras gástricas. Actualmente su uso se ha ampliado al ámbito gineco-obstétrico para lograr la maduración cervical y así inducir el trabajo de parto o facilitar la extracción de restos embrionarios y también para la interrupción temprana del embarazo.

Las propiedades del misoprostol se han ido conociendo a nivel de la población general, siendo cada día más común su uso como método abortivo. De acuerdo a una encuesta realizada en 7 hospitales de Río de Janeiro, aproximadamente el $57 \%$ de las mujeres que ingresaban por aborto incompleto admitieron haber usado misoprostol como método abortivo $(8,9)$. Pese a ser un fármaco bastante efectivo en terminar un embarazo, un $10-15 \%$ de las pacientes continúan con la gesta-

Tabla II

ANOMALÍAS EN EXTREMIDADES RELACIONADAS A USO DE MISOPROSTOL DURANTE LA GESTACIÓN

\section{Extremidades inferiores}

\begin{tabular}{lr}
\hline Tipo & $(\%)$ \\
Equinovaro & 80,7 \\
Mesomelia & 15,8 \\
Artrogriposis & 14,0 \\
Luxación de cadera & 10,5 \\
Bandas amnióticas & 8,8 \\
Sindactilia & 8,8 \\
Hipoplasia de uñas & 7,0 \\
Braquidactilia & 1,8
\end{tabular}

\section{Extremidades superiores}

Tipo

Mesomelia

46,4

Sindactilia

21,4

Bandas amnióticas $\quad 17,9$

Artrogriposis $\quad 17,9$

Hipoplasia de uñas $\quad 14,3$

Braquidactilia $\quad 7,1$

\begin{tabular}{ll} 
Polidactilia $\quad 3,6$ \\
\hline
\end{tabular} 
ción con alto riesgo de alteraciones congénitas fetales. Se han informado casos de anomalías congénitas asociadas al uso de misoprostol como método abortivo fallido. Dentro de las anomalías descritas, las principales alteraciones corresponden a extremidades y SNC (Tabla II). Las alteraciones del SNC más frecuentes son el compromiso de uno o más nervios craneales, especialmente el $\mathrm{VI}$ y el VII que en conjunto generan el síndrome de Moebius (10-16).

El misoprostol se ha relacionado en múltiples estudios al síndrome de Moebius (10,17-24). Se ha estudiado el mecanismo por el cual misoprostol pudiese causar el SM. Múltiples teorías han surgido pero la más convincente es aquella que sugiere que el misoprostol genera un efecto vasoconstrictor, que resulta en isquemia transitoria fetal afectando principalmente los núcleos de los NC abducens y facial. El principal mecanismo propuesto hace referencia a las contracciones uterinas intensas y prolongadas que generaría una reducción del flujo sanguíneo en ciertas áreas (por ejemplo: nervios craneales) y la restricción del crecimiento y movimiento fetal adecuado. En el caso de alteraciones de las extremidades, estas se generarían por este mecanismo mixto. Por tanto, dichas contracciones pueden potencialmente crear un trastorno vascular en la unidad feto-placentaria además de una restricción espacial (2124).

Aun cuando se han informado casos de malformaciones asociadas al uso de misoprostol en el primer trimestre de gestación, existen escasos reportes en la literatura internacional que avalen 0 propongan datos estadísticamente significativos para sustentar el rol teratogénico del misoprostol. Por esta razón es importante dar a conocer aquellos casos clínicos que avalen el rol teratogénico del misoprostol cuando es utilizado durante la etapa de organogénesis embrionaria.

\section{CONCLUSIÓN}

El caso presentado relaciona el uso de misoprostol durante el primer trimestre de la gestación con un cuadro de artrogriposis múltiple congénita y síndrome de Moebius al nacer. Se destaca la importancia del diagnóstico ultrasonográfico prenatal. Respecto al síndrome de Moebius se reporta un aumento de casos relacionados al uso de misoprostol durante el primer trimestre. Frente a esta situación, es cada vez más importante advertir a la comunidad médica y a la población en general de los efectos potencialmente teratogé- nicos de este fármaco. Por el aumento del uso de misoprostol como método abortivo es fundamental dar a conocer aquellos casos clínicos que avalen el rol teratogénico de este.

\section{BIBLIOGRAFÍA}

1. Jones KL. Artrogriposis Múltiple Congénita, Síndrome Moebius. En: Smith's Recognizable Patterns of Human Malformation. $4^{\mathrm{a}}$ ed. Philadelphia USA: editorial W.B. Sanders Company, 1988;142-3,1923.

2. Kleinsteuber K, Avaria MA. Enfermedades Neuromusculares en Pediatría. Rev Red Elec 2005;2(1): 52-61.

3. Hall JC. Genetics aspect of arthrogryposis. Clin Orthop 1985;194:44-53.

4. Kumar D. Moebius syndrome. J Med Genet 1990;27: 122-6.

5. Sarnat HB. Dysgenesis of the central nervous system. En: Principles of Child Neurology. Berg BO (ed). New York: McGraw-Hill 1996;629-49.

6. Downie WW. Misuse of misoprostol. Lancet 1991; 338(8761):247.

7. Misoprostol y teratogenicidad: Revisión de la evidencia. Informe de una reunión organizada por el Population Council New York, New York 22 de mayo de 2002. Hallado en: http://www.gynuity.org/ documents/miso_terat_sp.pdf

8. Costa SH, Vessey MP. Misoprostol and illegal abortion in Rio de Janeiro, Brazil. Lancet 1993; 341(8855):1258-61.

9. Osorio de Castro CGS, Pepe VLE, Luiza VL, Cosendey MAE, Freitas AM de, Miranda FF, et al. Uso indicado e uso referido de medicamentos durante a gravidez. Cad Saúde Publ 2004;20(Sup 1):S73-S82.

10. Gonzalez CH, Vargas FR, Perez AB, Kim AC, Brunoni D, Marques-Dias MJ, et al. Limb deficiency with or without Möbius sequence in seven Brazilian children associated with misoprostol use in the first trimester of pregnancy. Am J Med Genet 1993;47(1): 59-64.

11. Genest DR, Di Salvo D, Rosemblatt MJ, Holmes LB. Terminal transverse limb defects with tethering and omphalocele in a 17 week fetus following first trimester misoprostol exposure. Clin Dysmorphol 1999;8(1):53-8.

12. Hofmeyr GJ. Milos D, Nikodem VC, de Jager M. Limb reduction anomaly after failed misoprostol abortion. S Afr Med J 1998;88(5):566-7.

13. Gonzalez CH, Marques-Dias MJ, Kim CA, Sugayama SM, Da Paz JA, Huson SM, Holmes LB. Congenital abnormalities in Brazilian children associated with misoprostol misuse in first trimester of pregnancy. Lancet 1998;351(9116):1624-7.

14. Coelho KE, Sarmento MF, Veiga CM, Speck Martins CE, Safatle HP, Castro CV, Niikawa N. Misoprostol embryotoxicity: Clinical evaluation of fifteen patients 
with arthrogryposis. Am J Med Genet 2000;95(4): 297-301.

15. Paurngartten FJR, Magalhaes-de-Souza CA, de Carvalho RR, Chadoud I. Embryotoxic effects of misoprostol in the mouse. Braz J Med Biol Res 1995;28(3):355-61.

16. Schuler L, Pastuszak A, Sanseverino MT, Orioli IM, Brunoni D, Ashton-Prolla P, et al. Pregnancy outcome after exposure to misoprostol in Brazil- A prospective, controlled study. Reprod Toxicol 1999;13(2): 147-51.

17. Coelho HL. O que está em jogo no caso Cytotec. Ciência Hoje 1991;13(76):60-3.

18. Boudoux DD, Matos MAG, Gonçalves ED, Rocha M, Ventura LO, Hinrichsen SL. Síndrome de Moebius relacionada à ameaça de abortamento. Rev Bras Oftalmol 2000;59(3):173-7.

19. Vargas FR, Schuler-Faccini L, Brunoni D, Kim C, Meloni VFA, Sugayama SM, Albano L, Llerena JC Jr, Almeida JC, Duarte A, Cavalcanti DP, Goloni-
Bertollo E, Conte A,Koren G, Addis A. Prenatal exposure to misoprostol and vascular disruption defects: a case-control study. Am J Med Genet 2000;95(4): 302-6.

20. Fontenelle L, Araújo AP de QC, Fontana RS. Moebius syndrome: a case report. Arq Neuropsiquiatr 2001;59(3):812-4

21. Shepard TH. Moebius syndrome after misoprostol: a possible teratogenic mechanism. Lancet 1996;346: 780 .

22. Cavalcante R, Bezerra M, Giacheti C. Moebius syndrome related to misoprostol $\left(\operatorname{cytotec}^{\circledR}\right)$ use as an abortifacient RBPS 2005;18(3):140-4.

23. Oriofi IM, Castílla EE. Epidemiological assessment of misoprostol teratogenicity. BJOG 2000;107(4): 519-23.

24. Garamendi González PM, Landa Tabuyo MI. Misoprostol as an abortive in Spain: Report of a judicial autopsy case. Cuad Med Forense 2004;38:27-36. 